\title{
Los ladrillos del recinto amurallado de Talamanca de Jarama, Madrid: criterios para su diferenciación
}

\author{
R. FORT, M.J. VARAS, E. PÉREZ-MONSERRAT, J. LUQUE, M. ÁlVAREZ DE BUERGO, C. VÁZQUEZ-CALVO \\ Instituto de Geología Económica (CSIC-UCM), Facultad de CC. Geológicas \\ C/ José Antonio Novais, 2. Ciudad Universitaria. \\ 28040. Madrid. Spain.
}

\begin{abstract}
El recinto amurallado de Talamanca de Jarama tiene una larga historia llena de conflictos bélicos que lo han destruido en numerosas ocasiones. Por este motivo, la muralla ha sufrido numerosas etapas de reconstrucción y ampliación (s.IX, s.XIII, s.XIV y s.XVII), a las que hay que añadirle numerosas intervenciones de restauración en el último siglo (s.XX). Los ladrillos junto con los tapiales y las piedras son los principales materiales de construcción identificados.

Los ladrillos estudiados por microscopía de luz polarizada (MO), difracción de rayos X (DRX), espectroscolorometría, ultrasonidos y porosimetria de intrusión de mercurio, han permitido diferenciar cinco tipos, pertenecientes a distintas épocas de fabricación. Son los ladrillos más antiguos (tipo I) y los más modernos (tipo V), los que más diferencias muestran entre sí. En general, los cinco tipos de ladrillos presentan heterogeneidades debidas a una fabricación deficiente y que son las responsables del proceso de deterioro que les afecta, aunque estos ladrillos hayan sido colocados recientemente, en la última intervención.
\end{abstract}

Palabras clave: ladrillos históricos, recinto amurallado, técnicas no destructivas.

Bricks of the walled complex of Talamanca de Jarama, Madrid: Criteria for their differentiation

The walled complex of Talamanca de Jarama has a long history of wars that have destroyed it several times. Due to this fact, the Wall has gone through many reconstruction and enlargement processes $\left(9^{\text {th }}, 13^{\text {th }}, 14^{\text {th }}\right.$ and $17^{\text {th }}$ century), to which the restorations performed during the last century $\left(20^{\text {th }}\right)$ must be added. The main building materials are bricks together with rammed-earth and stones.

The bricks studied under Polarizing Microscopy (OM), X-ray powder diffraction (XRD), spectrocolourimetry, ultrasounds and mercury intrusion porosimetry (MIP), allowed us to distinguish five types, each of them belongs to different manufacturing periods. The oldest bricks (type I) and the most recent ones (type V), are those that differentiate the most. The five types of bricks show heterogeneities due to a deficient manufacture, which is responsible for their decay process, despite the fact that these bricks have been placed recently, during the last intervention.

Keywords: historic bricks, walled complex, non-destructive techniques.

\section{INTRODUCCIÓN}

El recinto amurallado de Talamanca de Jarama (Madrid) es un vestigio del patrimonio histórico con que cuenta la Comunidad de Madrid. La muralla fue construida posiblemente en épocas prerromanas, pero no adquiere importancia hasta la conquista árabe cuando el emir Muhammad I, hacia mediados del siglo IX, la convierte en ciudad-fortaleza. Al ser un bastión adelantado de las defensas de Madrid (Mayrit), Talamanca de Jarama sufre continuas contiendas que provocan daños de diversa envergadura en sus defensas (murallas). De este modo, se han podido constatar numerosos episodios de destrucción en los años 861, 878, 939, 1047-1050, y 1062 (1-2). En 1086 la localidad pasa definitivamente a manos cristianas. Hacia la primera mitad del siglo XIII (1217-1223), el arzobispo Jiménez de Rada reconstruye y amplia el recinto amurallado de Talamanca. A finales del siglo XIV, las murallas son otra vez ampliadas debido a los frecuentes ataques de los bandidos, y se eleva la altura de sus paramentos y torreones. En el siglo XVII se modificó el trazado de la muralla al construirse la Cartuja. En 1931 fue declarado Monumento HistóricoArtístico (1-5).

Las actuaciones más importantes fueron realizadas en 1982, con la consolidación y la reconstrucción de varias partes de la muralla destacando la Puerta de la Villa y la Puerta de Uceda (Fig. 1).

El recinto amurallado actual ocupa una superficie aproximada de 91 hectáreas y un perímetro de 1300 metros, semejante a un pentágono irregular. (Fig. 2). El trazado no se conserva en su totalidad, existiendo numerosos tramos donde la muralla ha desaparecido, principalmente en las zonas Oeste y Norte debido a la edificación de la Cartuja y a construcciones recientes. La historia de este recinto amurallado se refleja en la existencia de tres grandes fases constructivas que abarcan las etapas: árabe (s.IX), bajomedieval (principios del s.XIII) y altomedieval (finales del s.XIV). Estas tres fases constructivas 


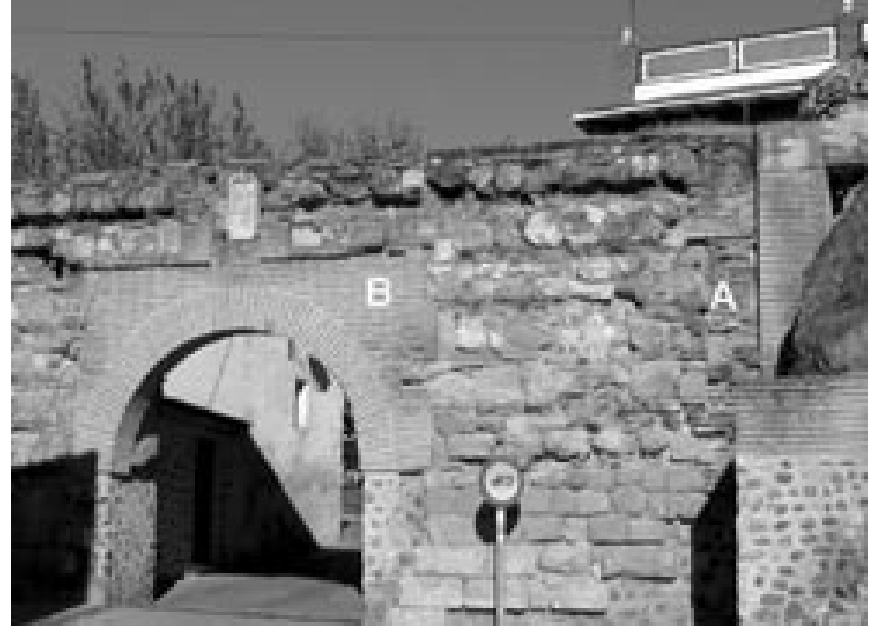

Fig. 1- Puerta de la Villa (s.XIII). Se encuentra muy restaurada con los ladrillos de tipo $\mathrm{V}(\mathrm{A})$, pero tiene zonas donde todavía existen ladrillos de tipo II (B).

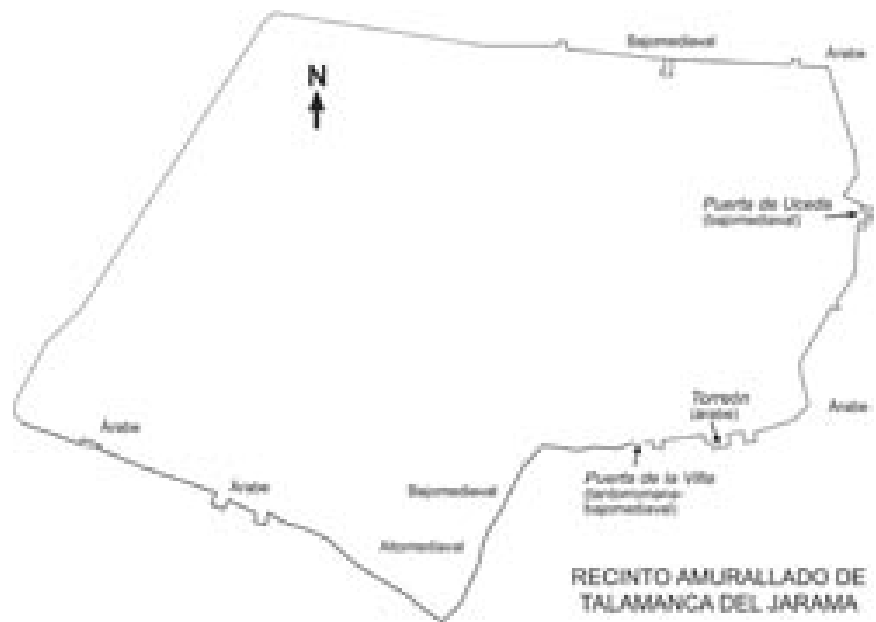

Fig. 2- Plano del recinto amurallado, dividido en distintos sectores (F) y con las tres fases constructivas bien identificadas.

aparecen intercaladas de tal modo que en muchas ocasiones no se pueden diferenciar claramente. La muralla está construida con tres tipos de fábrica, que se encuentran mezcladas: tapial, ladrillo, y mampuesto o sillarejo de piedra caliza.

Para este trabajo solo se ha elegido la fábrica de ladrillo, ya que el objetivo principal que se persigue es definir los diferentes parámetros, tanto físicos como químicos y mineralógicos, que nos permitan diferenciar estos ladrillos históricos en base a sus técnicas de fabricación y por consiguiente de sus etapas constructivas.

Los ladrillos históricos son materiales cerámicos obtenidos del amasado a mano, secado al sol y cocción posterior de materias primas arcillosas. Las temperaturas de cocción de estas materias primas arcillosas ha variado a lo largo de la historia entre 650 y $1000{ }^{\circ} \mathrm{C}$ (6). El color, la composición y las propiedades físicas y mecánicas de los ladrillos dependen de la naturaleza de la arcilla empleada (caolinita, illita, esmectita), del tipo de horno usado para su fabricación, de las condiciones reinantes dentro de los hornos (oxidante o reductor), de la temperatura y procesos de cocción sufridos, de la naturaleza y cantidad del desgrasante añadido (cuarzo, carbonatos, cerámica molida,...), etc (7). Bajo unas condiciones apropiadas, tanto la fase silícea como la fase carbonatada, que configuran las materias primas arcillosas de las que se parte, están sujetas a una serie de procesos térmicos dentro de los hornos (710): deshidratación de todos los componentes mineralógicos entre 100 y $400^{\circ} \mathrm{C}$, deshidroxilación de los silicatos entre 400 y $900^{\circ} \mathrm{C}\left(\mathrm{SiO}_{2^{\prime}} \mathrm{Al}_{2} \mathrm{O}_{3^{\prime}} \mathrm{MgO}\right.$ y $\left.\mathrm{Fe}_{2} \mathrm{O}_{3}\right)$ y descarbonatación de los materiales calcáreos entre 800 y $\left.1000^{\circ} \mathrm{C}(\mathrm{MgO}, \mathrm{CaO} \text { y CO})_{2}\right)$. A altas temperaturas de cocción $\left(>900^{\circ} \mathrm{C}\right)$, los productos de descomposición de los carbonatos reaccionan con los de las arcillas para formar nuevas fases mineralógicas; son principalmente silicatos cálcicos y / o alumínicos, tales como la gehlenita $\left(\mathrm{Ca}_{2} \mathrm{Al}_{2} \mathrm{SiO}_{7}\right)$, la wollastonita $\left(\mathrm{CaSiO}_{3}\right)$ y el diópsido $\left(\mathrm{CaMg}\left(\mathrm{SiO}_{3}\right)_{2}\right)$, y por encima de $\operatorname{los} 1100^{\circ} \mathrm{C}$ aparecen la mullita $\left(\mathrm{Al}_{6} \mathrm{SiO}_{13}\right)$ y la cristobalita $\left(\mathrm{SiO}_{2}\right)(10-16)$. En cualquier caso, nunca se supera la temperatura de fusión $\left(1300^{\circ} \mathrm{C}\right)$.

\section{METODO EXPERIMENTAL}

Para este estudio se recogieron 31 muestras de ladrillos procedentes de las diversas fases constructivas. La identificación de las diferentes tipologías de ladrillos se ha realizado por sus características petrográficas, mineralógicas y físicas. Las técnicas analíticas empleadas fueron, desde el punto de vista petrográfico y mineralógico, la mineralogía óptica de luz polarizada (Olympus BX51 con cámara Olympus DP12) y la difracción de rayos $X$ de la fracción polvo de roca total (Phillips PW-1752 con monocromador y ánodo de $\mathrm{Cu}$ ). Y desde el punto de vista físico, se analizó el color mediante el estudio de las coordenadas cromáticas $\mathrm{L}^{*}, \mathrm{a}^{*} \mathrm{~b}^{*}$ y $\mathrm{C}^{*}$ con un espectrocolorímetro Minolta CM 2002 (17), la velocidad de propagación de ultrasonidos y el grado de anisotropía con un "PUNDIT, CSN Electrinic" y la distribución del tamaño de los poros mediante la realización de porosimetrías de intrusión de mercurio (Micromeritics Autopore IV-9520).

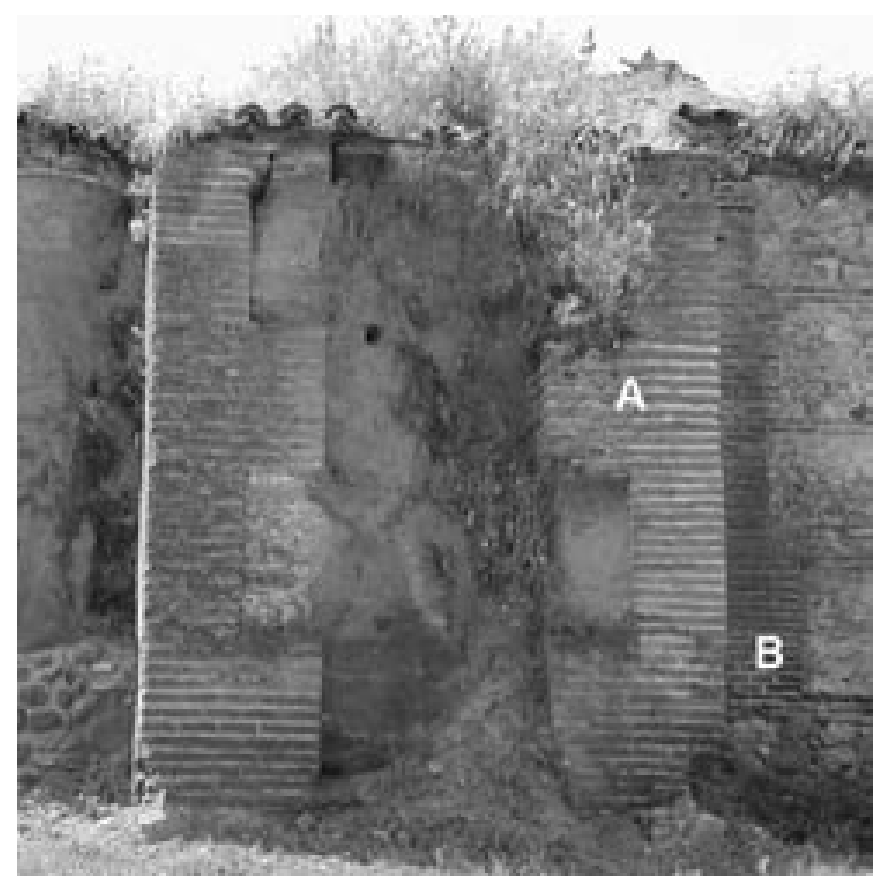

Fig. 3- Sector antiguo (s.IX) de la muralla localizado cerca de la Cartuja (s.XVII). Aunque los tapiales aparecen muy deteriorados, los ladrillos dominantes son del tipo I (A), habiendo zonas donde se han reconocido ladrillos del tipo IV (B). 


\section{RESULTADOS Y DISCUSIÓN}

\subsection{Dimensiones de los ladrillos}

En la muralla se han podido distinguir cinco tipos de ladrillos, en base a sus dimensiones. Puede indicarse que los ladrillos más grandes (tipo I; Tabla 1), se localizan en los muros que aparecen al Norte de la villa (Fig. 3). Los ladrillos de la Puerta de la Villa y del muro de contención adyacente (F2) (Figs 1, 2 y 4), presentan unas dimensiones muy diferentes, dominando los de tipo II. En este último paramento (F2) predomina el ladrillo como material de construcción, existiendo en las hiladas superiores otro tipo de ladrillo con dimensiones ligeramente inferiores en la soga y que podrían ser ladrillos de tipo III (Fig. 4).

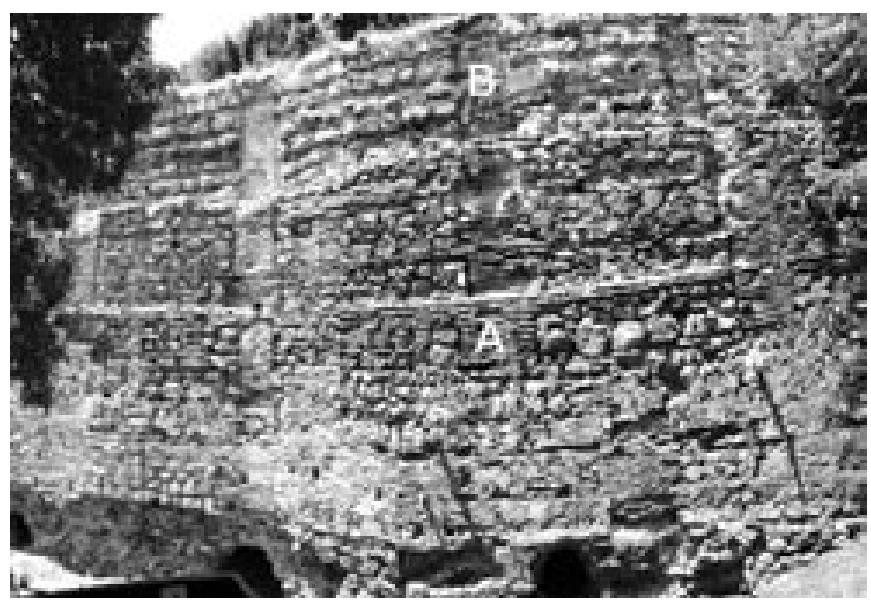

Fig. 4- Sector de la muralla (F2) que se encuentra en buen estado de conservación (s.XIII). En esta zona se identifican principalmente ladrillos del tipo II (A), aunque en la parte superior se reconoce el añadido de los ladrillos de tipo III (B) puestos en obra durante la fase de elevación en altura de la muralla (s.XIV).

Los ladrillos del tipo III presentan una soga ligeramente inferior y aparecen en sobre todo en zonas donde dominan los restos de muralla. Este tipo también destaca en las zonas altas y medias del Torreón (F1), localizado a la derecha de la Puerta de la Villa, en la zarpa del sector F3 y sector F5 (Fig. 5).

Este tipo III de ladrillo, debió de colocarse en las importantes actuaciones de restauración y elevación de la muralla llevadas a cabo en el siglo XIV, que están bien documentadas por los antecedentes históricos. En esa época, también se realizaron arreglos en la parte alta de la Puerta de la Villa, se construyó o reforzó otra zarpa existente al Sur (F3), y se amplió o reconstruyó en altura del Torreón existente en el sector F1 (Fig. 5).

Losladrillos de tipo IV, fueron utilizadosen reconstrucciones relativamente recientes, localizándose principalmente en la cerca y paramentos de la Cartuja (s.XVII) (Fig. 3), y en las zonas altas de los restos de muralla existentes en el sector F1 (Fig. 4).

TABLA 1. DIMENSIONES DE LOS CINCO TIPOS DE LADRILLOS IDENTIFICADOS (cm)

\begin{tabular}{|c|c|c|c|}
\hline TIPO & SOGA & TIZÓN & GRUESO \\
\hline V & 23,05 & 10,77 & 3,68 \\
\hline IV & $25,94 \pm 0,42$ & $14,37 \pm 1,95$ & $4,30 \pm 0,42$ \\
\hline III & $27,03 \pm 0,49$ & $16,81 \pm 0,83$ & $4,15 \pm 0,36$ \\
\hline II & $28,93 \pm 0,69$ & $16,59 \pm 0,67$ & $4,56 \pm 0,32$ \\
\hline I & $33,61 \pm 0,69$ & $21,43 \pm 0,78$ & $5,27 \pm 0,35$ \\
\hline
\end{tabular}

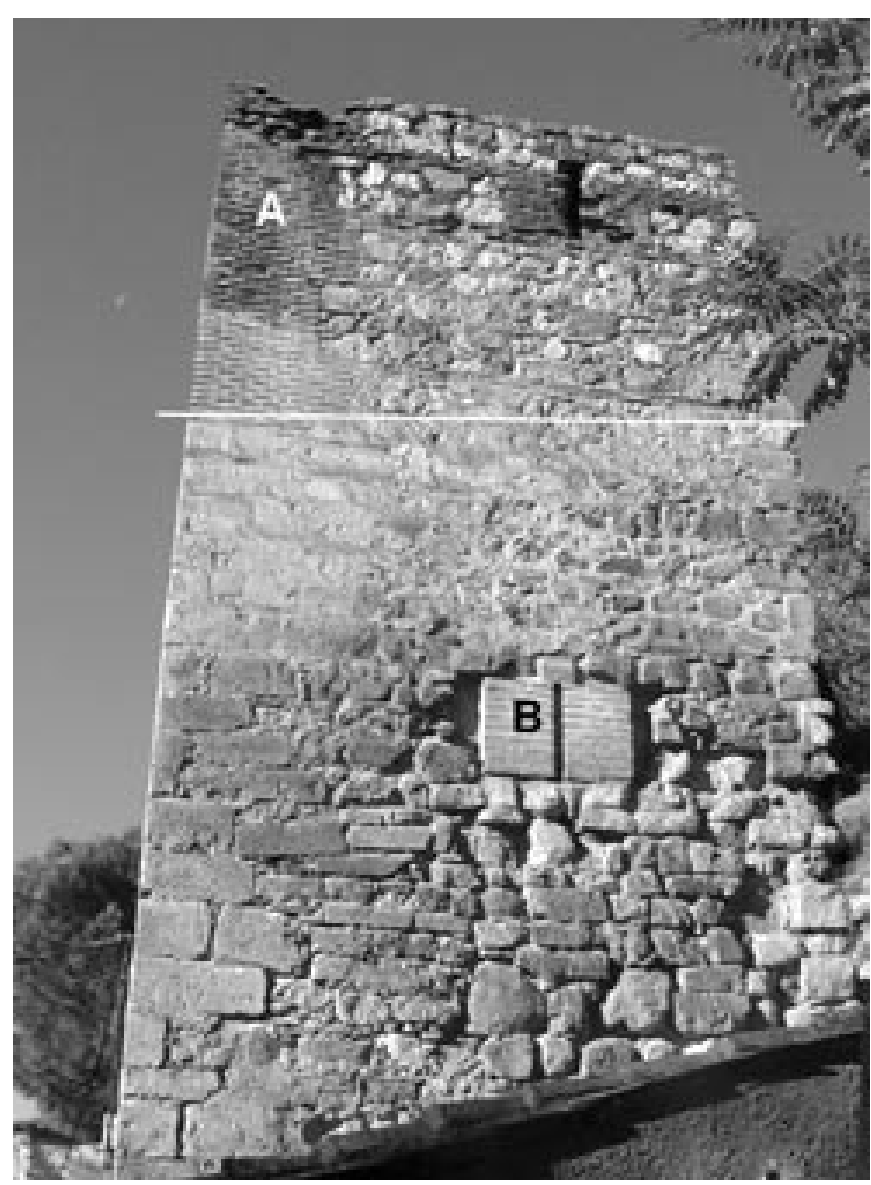

Fig. 5- Torreón (F1) donde la elevación de sus paramentos ha implicado la colocación de ladrillos de tipo III, durante el s.XIV. En las intervenciones de restauración llevadas a cabo durante el s.XX, se añadieron localmente ladrillos del tipo $\mathrm{V}(\mathrm{B})$.

Por último, los ladrillos con menores dimensiones, tanto en soga como en tizón y grueso (tipo V), fueron utilizados en las últimas actuaciones acontecidas en la muralla, y llevadas a cabo en el siglo XX. Se localizan en muchos paramentos de la muralla, principalmente en las zarpas del sector F3, en la Puerta de la Villa y en algunas zonas de las arpilleras del Torreón del sector F1 (Fig. 1, 2 y 5).

Los ladrillos han podido ser reutilizados en diferentes épocas, y por ese motivo no será válida la utilización aislada de esta técnica para la datación de las fases constructivas de los paramentos de esta muralla, sino que tendrá que ir acompañada de una lectura más detallada de la disposición de estos elementos en cada paramento.

\subsection{Petrología y mineralogía}

Las características petrográficas de los cinco tipos de ladrillos son muy similares. Los áridos están constituidos por cuarzo, feldespato potásico y fragmentos de roca de origen granítico y/o metamórfico, identificándose en algunas piezas pellas carbonáticas y/o arcillosas, y en otras, abundantes filosilicatos, como moscovitas y biotitas. Además presentan una porosidad característica orientada paralelamente a la superficie de los ladrillos.

Las diferencias están marcadas por una mayor homogenidad y compacidad en los ladrillos de tipo I (Fig. 6a), con un tamaño de árido más pequeño y menor porosidad que en las otras dos variedades. Sus áridos presentan morfologías 

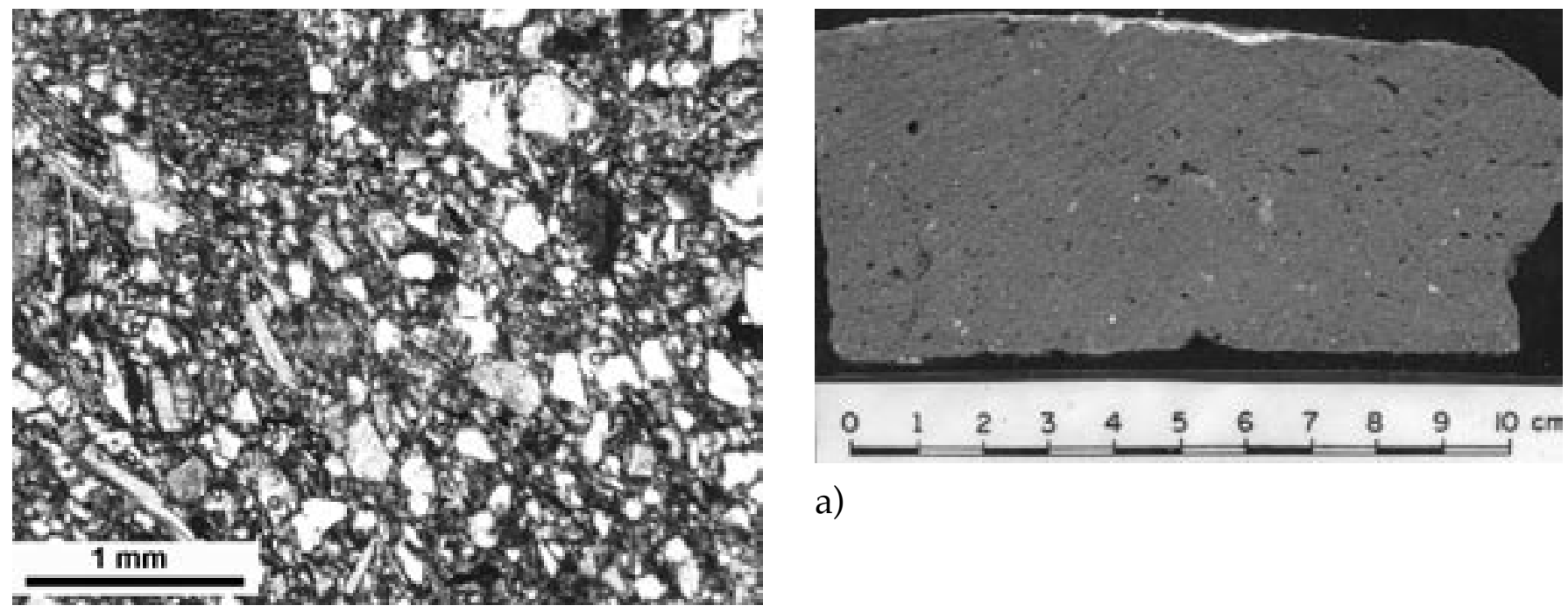

a)
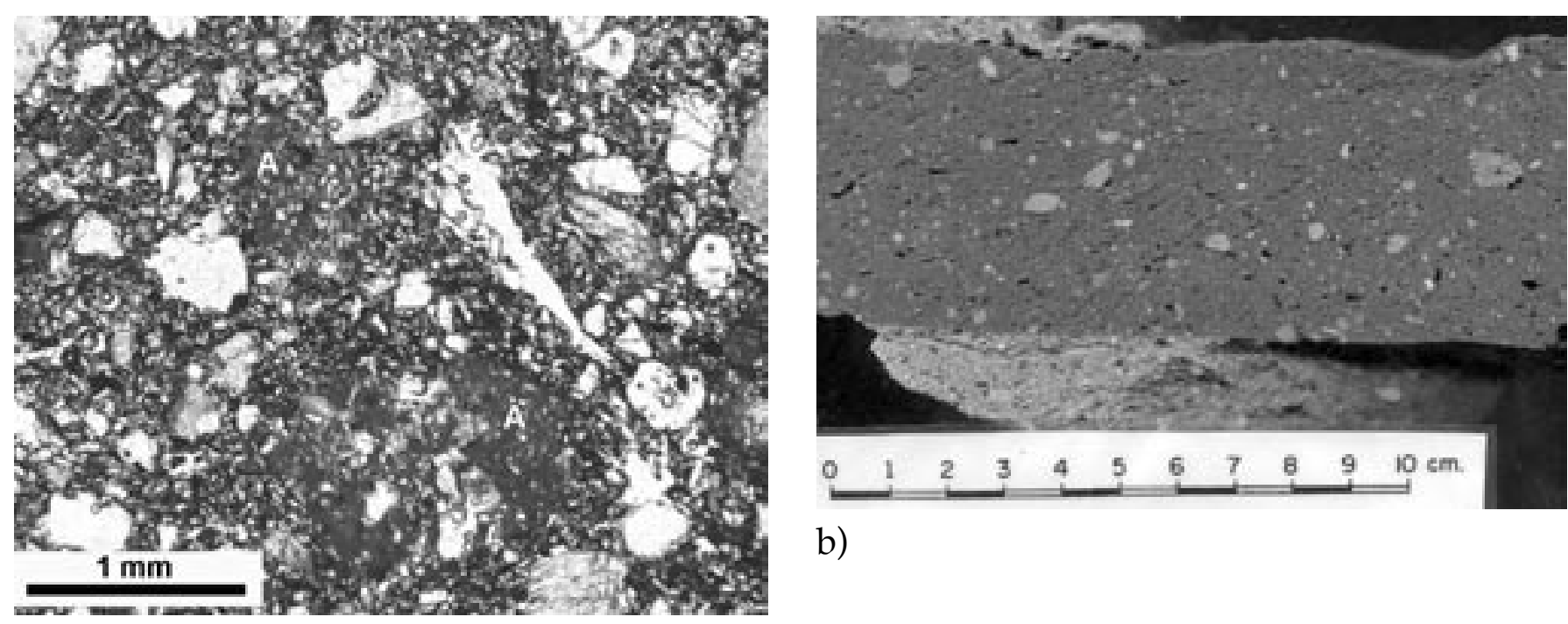

b)
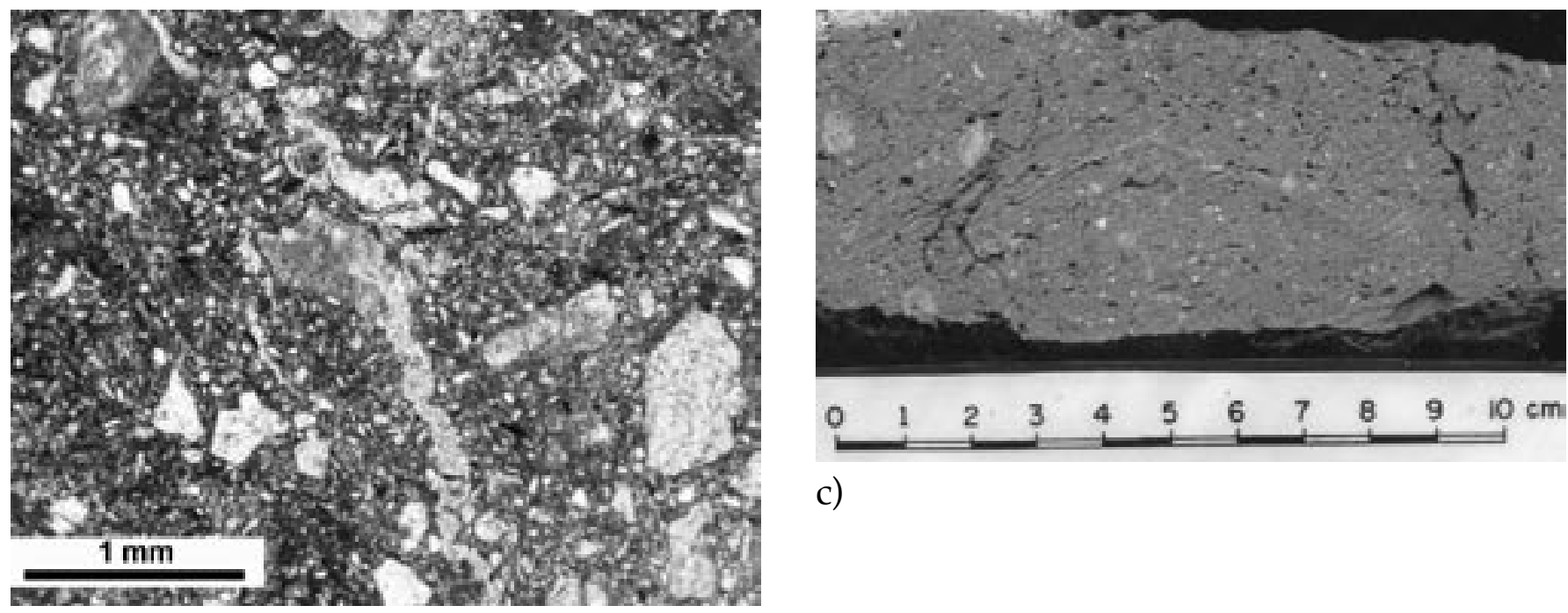

c) 

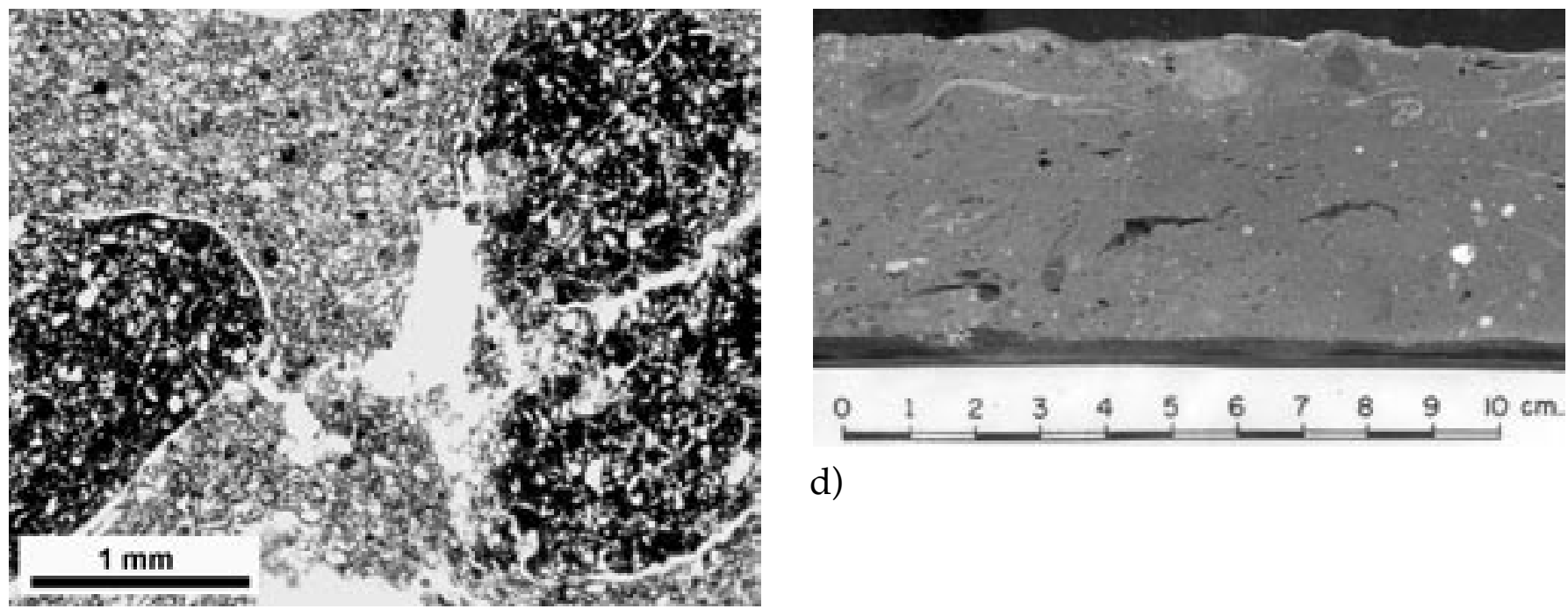

d)
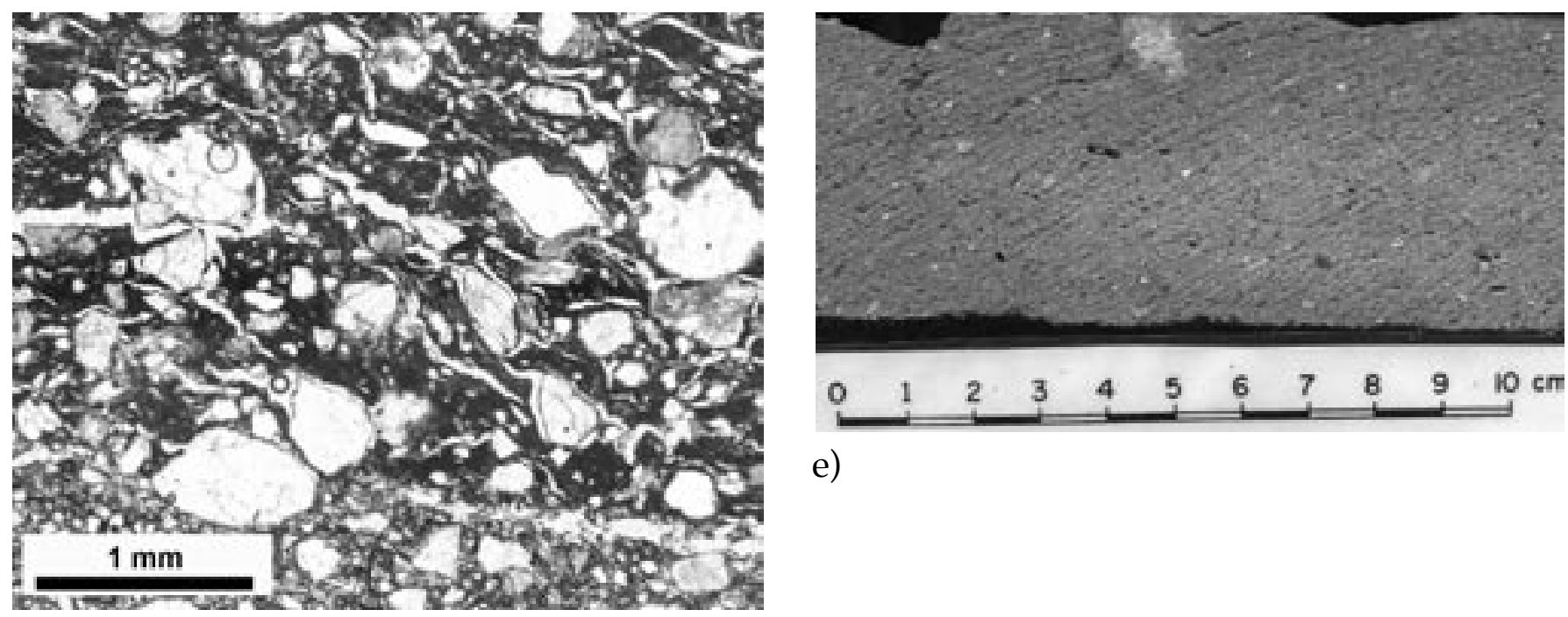

e)

Fig. 6- Aspecto visual y al microscopio petrográfico (luz natural) de los cinco tipos de ladrillos identificados en la muralla de Talamanca. a) Tipo I (más antiguo; alto empaquetamiento), b) Tipo II (A-pellas de cal), c) Tipo III, d) Tipo IV (pellas de arcilla) y e) Tipo V (más moderno; alto agrietamiento de la matriz ferruginosa).

angulosas-subangulosas y los de mayor tamaño morfologías más subredondeadas (Fig. 6a). Los ladrillos de tipo II se caracterizan por tener un gran número de pellas carbonáticas (cal) y mayor porosidad (Fig. 6b). Aunque su árido es subanguloso, los poros presentan morfologías muy variables. En el ladrillo de tipo III (Fig. 6c), el árido suele ser anguloso y los poros también presentan morfologías muy variables, disponiéndose algunos según una textura fluidal, originada durante el amasado de las piezas. Además de presentar pellas carbonáticas, este último tipo también presenta pellas de arcilla. Todas estas pellas presentan grietas de retracción a su alrededor, originadas por la contracción de estas masas carbonatadas y arcillosas durante la fase de cocción de las piezas.

Los ladrillos de tipo de II y III, además aparecen zonados, con cambios en el color hacia el interior del ladrillo. La pasta de algunas de estas piezas presenta en sus bordes una tonalidad más oscura, debido tal vez a la posición que ocupaban en el hormo durante su cocción.

Los ladrillos del tipo IV son diferentes a los anteriores tipos. Sus áridos son principalmente de cuarzo y fragmentos de roca graníticas, junto con fermentos de rocas metamórficas y de caliza. Existen numerosas pellas arcillosas con fisuración interna y grietas de retracción a su alrededor (Fig. 6d). Este tipo de ladrillos también aparecen zonados, presentando numerosas bandas de cocción (coloración diferencial).

Los ladrillos más modernos (tipo V) tienen áridos subangulosos de cuarzo, siendo los de mayor tamaño más subredondeados, fragmentos de roca graníticas, feldespatos potásicos, biotitas y bastantes minerales opacos. La matriz de estos ladrillos puede llegar a estar muy agrietada y oxidada (Fig. 6e).

Las fases mineralógicas identificadas mediante el análisis de difracción de rayos $X$ en estos ladrillos son principalmente cuarzo, y en cantidades menores: micas, microclinas y plagioclasas, arcillas, calcita, dolomita, hematites, gehlenita y diópsido. Los hematites, la gehlenita y el diópsido, son fases mineralógicas secundarias, aparecidas a temperaturas de $900^{\circ} \mathrm{C}$ durante la cocción de los ladrillos en los hornos (10-16). El resto de fases minerales son primarias.

Los ladrillos del tipo I y III se caracterizan por tener una alta concentración en micas, que junto con la presencia de dolomita, indican temperaturas de cocción inferiores a los $900^{\circ} \mathrm{C}(10-16)$. La temperatura de $900^{\circ} \mathrm{C}$ queda confirmada por 
la presencia del diópsido y la gehlenita. Al mismo tiempo, presentan pequeñas cantidades de arcilla esmectítica, lo que refleja una cocción irregular de las piezas, no llegando a superar los $600^{\circ} \mathrm{C}$ de temperatura en su interior y los $700^{\circ} \mathrm{C}$ en su superficie (10-16). Así pues, las temperaturas de cocción para estos ladrillos estarían comprendidas entre los 700 y los $900{ }^{\circ} \mathrm{C}$.

Los ladrillos del tipo II no tienen micas por lo que su temperatura de cocción es superior a los $900^{\circ} \mathrm{C}$. Esta temperatura también queda confirmada por la presencia de óxidos de hierro (hematites), diópsido y gehlenita que aparecen en torno a estos $900^{\circ} \mathrm{C}$ (10-16). Al mismo tiempo, la existencia de calcita indica que la temperatura de cocción no supera $\operatorname{los} 1000^{\circ} \mathrm{C}$, ya que es a partir de esta temperatura cuando la descomposición de los materiales carbonáticos es completa (18). La temperatura de cocción para este tipo de ladrillos es alta, rondando los $900-1000^{\circ} \mathrm{C}$.

Los ladrillos del tipo IV y V se asimilan en las temperaturas de cocción porque presentan una mineralogía muy similar. Las micas son escasas por lo que la temperatura de cocción podía rondar los $900^{\circ} \mathrm{C}$. Junto con la presencia de óxidos de hierro (hematites) y diópsido, se confirma una temperatura de cocción entre los 800 y $900^{\circ} \mathrm{C}(10-16)$. En el caso, de los ladrillos de tipo IV la presencia de algunas arcillas indica que existe una cocción irregular o al menos poco uniforme en su interior, no llegando a superar en algunos puntos $\operatorname{los} 600^{\circ} \mathrm{C}$, aunque la temperatura de cocción general sea de $800-900^{\circ} \mathrm{C}$. En el caso de los ladrillos de tipo $\mathrm{V}$, la cocción es más uniforme.

\subsection{Medidas de Color}

Los valores cromáticos obtenidos para los cinco tipos de ladrillos (Tabla 2) son muy similares, lo que indica una cuidada selección y mezcla de la pasta arcillosa a la hora de fabricar los ladrillos de sustitución, de modo que guarden una alta similitud cromática con los originales. Las coordenadas cromáticas $\mathrm{a}^{*} \mathrm{y} \mathrm{b}^{*}$ son positivas, lo que indica una coloración que oscila entre el amarillo y el rojo, con mayor dominio de este último.

Los ladrillos más modernos de tipo $\mathrm{V}$, son algo más claros ( $\mathrm{L}^{*}$ alto) que los restantes y tienen un grado de saturación menor ( $\mathrm{a}^{*}, \mathrm{~b}^{*}$ y $\mathrm{C}^{*}$ bajos; Tabla 2). Del mismo modo, son los que mayor dispersión de valores presentan (alta desviación estándar). Los ladrillos originales de tipo I son bastante homogéneos, siendo junto con los ladrillos de tipo II los más oscuros ( $\mathrm{L}^{*}$ bajo), rojos (a* alto) y con mayor intensidad de color $\left(\mathrm{C}^{*}\right.$ alto). Esto indica que desde el punto de vista cromático, los ladrillos de tipo I y II son muy similares, diferenciándose claramente de los de tipo V (Tabla 2). Los ladrillos de tipo

TABLA 2. PARÁMETROS CROMÁTICOS DE LOS LADRILLOS

\begin{tabular}{|c|c|c|c|c|c|c|c|c|}
\hline & \multicolumn{2}{|c|}{ L $^{*}$} & \multicolumn{2}{c|}{$b^{*}$} & \multicolumn{2}{c|}{$\mathrm{a}^{*}$} & \multicolumn{2}{c|}{$\mathrm{C}^{*}$} \\
\hline TIPO & Media & Std & Media & Std & Media & Std & Media & Std \\
\hline V & 57,35 & 5,43 & 12,55 & 6,82 & 22,96 & 3,79 & 26,46 & 6,66 \\
\hline IV & 50,45 & 4,45 & 19,50 & 4,97 & 27,63 & 2,76 & 33,86 & 5,12 \\
\hline III & 50,32 & 5,65 & 17,72 & 3,46 & 21,46 & 4,73 & 27,95 & 5,16 \\
\hline II & 47,33 & 5,24 & 19,71 & 3,15 & 24,87 & 3,00 & 31,83 & 3,44 \\
\hline I & 47,82 & 2,65 & 17,54 & 1,73 & 24,54 & 1,90 & 30,20 & 2,00 \\
\hline
\end{tabular}

$\mathrm{L}^{*}$ : Luminosidad; $\mathrm{a}^{*}$ : tonos rojos $(+\mathrm{a})$ y verdes $(-\mathrm{a}) ; \mathrm{b}^{*}$ : tonos amarillo $(+\mathrm{b}) \mathrm{y}$ azul(-b); $C^{*}$ : Croma. Media-Valores medios y Std-Desviación estandar.
III y IV, aunque presentan una luminosidad similar difieren ligeramente en el resto de parámetros cromáticos. El tipo IV comprende los ladrillos con mayor intensidad de color $\left(\mathrm{C}^{*}\right.$ $\approx 34)$, siendo el rojo el color dominante $\left(a^{*} \approx 27,6\right.$; Tabla 2$)$.

\subsection{Ultrasonidos y anisotropía}

La tabla 3, incluye los valores medios de los ladrillos recogidos en diferentes puntos de la muralla. Puede apreciarse que la menor velocidad de propagación de ultrasonidos corresponde a los ladrillos de sustitución empleados en la última restauración (tipo V). Junto a los altos índices de anisotropía que presentan, estos ladrillos son un reflejo de la presencia de heterogeneidades, que pueden ser debidas a los procesos de fabricación, y favorecer así los procesos de degradación. Esto índica que este tipo de ladrillo tiene una alta porosidad que hace que los procesos de deterioro sean importantes en él, aunque haya pasado un corto periodo de tiempo desde su puesta en obra. Los ejemplares originales también presentan una baja velocidad de propagación de ultrasonidos, que puede ser reflejo de sus diferentes grados de alteración. Del mismo modo, los de tipo I tienen un índice de anisotropía media muy bajo. El índice de anisotropía $\Delta \mathrm{M}$ es el que mejor diferencia cada tipología, ya que existe una

TABLA 3. VELOCIDAD DE PROPAGACIÓN DE ULTRASONIDOS E ÍNDICES DE ANISOTROPÍA

\begin{tabular}{|c|c|c|c|c|c|c|}
\hline \multirow{2}{*}{ TIPO } & \multicolumn{2}{|c|}{$\mathrm{Vp}(\mathrm{m} / \mathrm{s})$} & \multicolumn{4}{|c|}{ Indice de Anisotropía } \\
\cline { 2 - 7 } & \multicolumn{2}{|c|}{} & \multicolumn{2}{|c|}{$\Delta \mathrm{M} \%$} & \multicolumn{2}{c|}{$\Delta \mathrm{m} \%$} \\
\cline { 2 - 7 } & Media & Std & Media & Std & Media & Std \\
\hline V & 1596 & 210 & 19,4 & 8,1 & 9,8 & 5,3 \\
\hline IV & 2187 & 380 & 15,4 & 5,1 & 10,4 & 5,9 \\
\hline III & 2517 & 944 & 11,8 & 6,56 & 6,4 & 5,4 \\
\hline II & 2338 & 498 & 18,3 & 11,40 & 8,1 & 5,8 \\
\hline I & 1862 & 538 & 9,8 & 6,89 & 5,6 & 4,1 \\
\hline
\end{tabular}

Vp- Velocidad de propagación de ultrasonidos. $\Delta \mathrm{M}$-Índice de anisotropía total. $\Delta \mathrm{m}$-Índice de anisotropía relativa. Media-Valores medios y Std-Desviación estándar.

tendencia relativa a incrementar su valor desde el tipo I al de tipo $\mathrm{V}$, tal como se ve en la tabla 3.

\subsection{Densidades y Compacidad}

La densidad real de los ladrillos utilizados en las diferentes fases de reconstrucción de la muralla varía de 2,57 a 2,63 $\mathrm{g} / \mathrm{cm}^{3}$. Por el contrario, la densidad aparente es $1,65-1,77$ $\mathrm{g} / \mathrm{cm}^{3}$. La alta diferencia que hay con la densidad real indica una presencia elevada de porosidad. En la tabla 4 se muestra el valor medio para cada uno de los tipos de ladrillos. Puede apreciarse cómo no existen grandes diferencias dadas las desviaciones estándar que tienen cada una de las poblaciones

TABLA 4. DENSIDAD Y COMPACIDAD DE LOS LADRILLOS

\begin{tabular}{|c|c|c|c|c|c|c|}
\hline & \multicolumn{2}{|c|}{$\begin{array}{c}\text { Densidad real } \\
\left(\mathrm{g} / \mathrm{cm}^{3}\right)\end{array}$} & \multicolumn{2}{|c|}{$\begin{array}{c}\text { Densidad aparente } \\
\left(\mathrm{g} / \mathrm{cm}^{3}\right)\end{array}$} & \multicolumn{2}{|c|}{$\begin{array}{c}\text { Índice } \\
\text { Compacidad } \\
(\%)\end{array}$} \\
\hline TIPO & Media & Std & Media & Std & Media & Std \\
\hline V & 2,605 & 0,049 & 1,726 & 0,046 & 65,31 & 1,61 \\
\hline IV & 2,582 & 0,060 & 1,649 & 0,078 & 63,88 & 1,90 \\
\hline III & 2,575 & 0,072 & 1,775 & 0,112 & 68,97 & 4,31 \\
\hline II & 2,577 & 0,094 & 1,765 & 0,064 & 68,66 & 4,09 \\
\hline I & 2,627 & 0,056 & 1,735 & 0,049 & 66,06 & 2,56 \\
\hline
\end{tabular}


consideradas, pero donde destacan los dos tipos de ladrillos más modernos (tipos IV y V), seguidos de cerca por el tipo I, más antiguo. En estos tres casos, las diferencias entre ambas densidades son ligeramente mayores, marcando una porosidad algo más alta.

\subsection{Porosidad y distribución de tamaños de poros}

Con respecto a la porosidad accesible al agua, los ladrillos más modernos, correspondientes a los tipos IV y V, son los que tienen valores más altos, seguidos de cerca por los ladrillos originales del tipo I (Tabla 5).

TABLA 5. POROSIDAD ACCESIBLE AL AGUA Y CANTIDAD DE AGUA QUE PUEDEN CONTENER LOS LADRILLOS

\begin{tabular}{|c|c|c|c|c|}
\hline & \multicolumn{2}{|c|}{$\begin{array}{c}\text { Porosidad accesible al agua } \\
(\%)\end{array}$} & \multicolumn{2}{c|}{$\begin{array}{c}\text { Saturación al agua } \\
(\%)\end{array}$} \\
\hline TIPO & Media & Std & Media & Std \\
\hline V & 34,71 & 1,60 & 19,81 & 1,87 \\
\hline IV & 36,12 & 1,72 & 21,90 & 1,95 \\
\hline III & 31,03 & 4,31 & 17,66 & 3,51 \\
\hline II & 31,34 & 4,09 & 17,87 & 2,83 \\
\hline I & 33,94 & 2,56 & 19,61 & 2,04 \\
\hline
\end{tabular}

En la tabla 6 aparecen reflejados los datos obtenidos en el ensayo de porosimetría de mercurio. Estos datos son de gran interés para saber el comportamiento de estos materiales ante los procesos de alteración, ya que el sistema poroso de los ladrillos, y sobre todo su distribución porosimétrica, son los que controlan la entrada del agua y de los agentes de deterioro hacia el interior de cada pieza cerámica $(19,20)$.

Losladrillos detipoIse caracterizan por tener una porosidad media de $33,4 \%$, donde predomina la microporosidad sobre la macroporosidad (Tabla 6). La distribución de tamaños de poros es unimodal, concentrándose la mayoría de los poros existentes en estos ladrillos en torno a 1,5 $\mu \mathrm{m}$ (Fig. 7). También es destacable el reducido tamaño de los poros $(0,18$ $\mu \mathrm{m})$ frente a su elevada superficie específica $\left(7,3 \mathrm{~g} \cdot \mathrm{m}^{-2}\right)$. Los

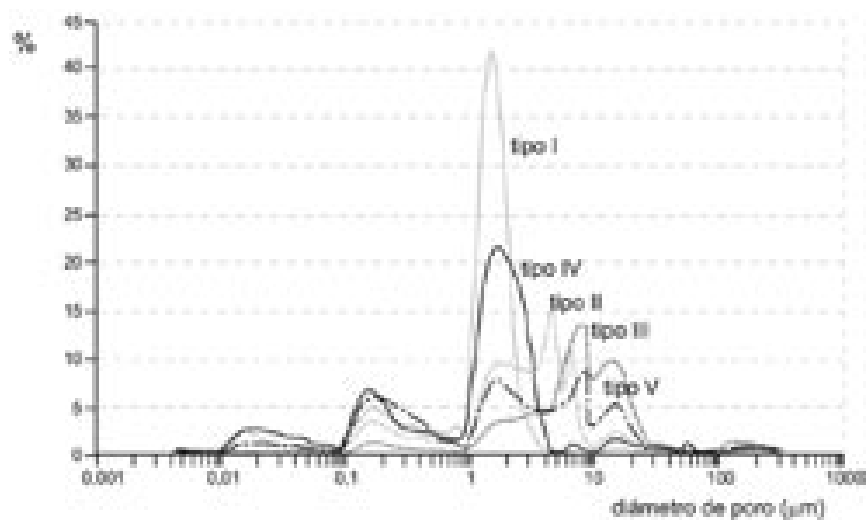

Fig. 7- Distribución porosimétrica de los cinco tipos de ladrillos. del tipo II tienen la porosidad más baja de todos, aunque sus poros son los de mayor tamaño medio $(2,81 \mu \mathrm{m})$ y menor superficie específica (4,43 g.m $\left.\mathrm{m}^{-2}\right)$ (Tabla 6). En estos ladrillos, la distribución de la porosidad está marcada por un incremento de la macroporosidad. Su distribución porosimétrica es polimodal, concentrándose principalmente en el intervalo 1 a $10 \mu \mathrm{m}$, donde existen tres máximos a 1,5, 4 y $6 \mu \mathrm{m}$ (Fig. 7).

Los ladrillos tipo III, tienen una porosidad similar a la de los del tipo I, pero con un porcentaje muy parecido tanto en microporosidad como en macroporosidad (Tabla 6). La distribución de los tamaños de poros presenta un sesgo hacia diámetro de poros mayores (macroporosidad), con una mediana de la distribución cercana a las $4 \mu \mathrm{m}$. Este tipo de ladrillos presenta dos modas importantes a $7 \mu \mathrm{m}$ y a $15 \mu \mathrm{m}$.

Los ladrillos analizados del tipo IV se asimilan a los del tipo I, mostrando un claro dominio de la microporosidad frente a la macroporosidad (Tabla 6). También es destacable el pequeño tamaño de sus poros frente a su elevada superficie específica (Tabla 6). La distribución porosimétrica también es unimodal, en torno a $2 \mu \mathrm{m}$, siendo importante también la moda localizada entre 0,1 y $0,2 \mu \mathrm{m}$ (Fig. 7).

Por último, los ladrillos de restauración pertenecientes al tipo $\mathrm{V}$, presentan unas características diferentes a todos los anteriores, con una porosidad más alta (>36\%), y una relación microporosidad/macroporosidad similar; tal y como se aprecia en la tabla 6. La distribución de tamaños de poros es polimodal con dos modas importantes en el intervalo 1-10 $\mu \mathrm{m}(1,5$ y $8 \mu \mathrm{m})$ y otras dos localizadas en los intervalos $0,1-0,4$ $\mu \mathrm{m}$ y $15 \mu \mathrm{m}$.

\section{CONCLUSIONES}

- En el recinto amurallado de Talamanca de Jarama se han detectado tres grandes fases constructivas que abarcan la etapa árabe (s.IX), bajomedieval (principios del s.XIII) y altomedieval (finales del s.XIV).

- Los ladrillos se agrupan en cinco tipos, tres de ellos (tipos I, II y III) corresponden a las tres etapas constructivas originales de la muralla (s.IX, s.XIII y s.XIV), otro tipo (tipo IV) a construcciones llevadas a cabo posteriormente (Cartuja y su entorno, s.XVII) y el tipo V corresponde a los ladrillos de sustitución colocados en diversos puntos de la muralla durante las intervenciones llevadas a cabo en el siglo XX.

- Las dimensiones de los ladrillos, la temperatura de cocción, estimada por la composición mineralógica de los ladrillos y, principalmente, la distribución de tamaños de los poros de los ladrillos, son los mejores criterios para diferenciar los distintos tipos de ladrillos existentes en la muralla, y que corresponden a diferentes épocas de construcción. La velocidad de propagación de ultrasonidos y los índices anisotropía de los ladrillos, así como la tonalidad cromática, también aportan alguna información de interés sobre los diferentes ladrillos utilizados.

TABLA 6. PARÁMETROS POROMÉTRICOS DE LOS DIFERENTES TIPOLOGÍAS DE LADRILLOS

\begin{tabular}{|c|c|c|c|c|c|c|}
\hline TIPO & (\%) Porosidad & $<5 \mu \mathrm{m}(\%)$ & $>5 \mu \mathrm{m}(\%)$ & $\begin{array}{l}\text { Tamaño medio de } \\
\text { poros }(\mu \mathrm{m})\end{array}$ & Mediana $(\mu \mathrm{m})$ & $\begin{array}{c}\text { Superficie específica } \\
\left(\mathrm{g} / \mathrm{m}^{2}\right)\end{array}$ \\
\hline $\mathrm{V}$ & 36,7 & 20,5 & 16,3 & 0,28 & 3,91 & 3,25 \\
\hline IV & 32,8 & 28,8 & 4,0 & 0,09 & 1,34 & 8,92 \\
\hline III & 32,5 & 18,6 & 13,4 & 0,69 & 4,37 & 3,04 \\
\hline II & 30,9 & 21,2 & 10,1 & 2,81 & 3,71 & 4,43 \\
\hline I & 33,4 & 28,8 & 4,5 & 0,18 & 2,56 & 7,31 \\
\hline
\end{tabular}


- Los resultados obtenidos muestran que los cinco tipos de ladrillos son similares entre sí, aunque existen claras diferencias entre ellos, sobre todo entre los ladrillos más antiguos (tipo I) y los más modernos (tipo V). Todo esto indica que son ladrillos con heterogeneidades en su interior que facilitan su degradación. Esta degradación afecta antes a los ladrillos más modernos que a los más antiguos. En todos los tipos de ladrillos analizados existe una buena selección de las materias primas, pero su fabricación es deficiente, falla la mezcla y el amasado, y su cocción en general es heterogénea.

- Los ladrillos de tipo I, más antiguos, son los de mayor tamaño, frente a los más modernos de tipo $\mathrm{V}$, que son los más pequeños.

- La petrología de los cinco tipos de ladrillos es muy similar. Son ladrillos con poco contenido en carbonatos, sus áridos son mineralógicamente similares, y la mayoría de los cinco tipos presentan una porosidad orientada paralelamente a la superficie de los ladrillos y una zonación, con cambios de color hacia el interior de los ladrillos. Todo esto indica poco cuidado en su fabricación (amasado deficiente y cocción heterogénea).

- Las diferencias texturales son más notorias y estriban en la gran homogeneidad de los ladrillos de tipo I; en la presencia de pellas de cal en los del tipo II; pellas de cal y arcilla en los del tipo III; pellas de arcilla en los del tipo IV y en la presencia de bastantes minerales opacos y grietas en los del tipo V.

- Atendiendo a su paragénesis mineral, estos cinco tipos de ladrillos se agrupan en tres, con tres rangos de temperatura de cocción similares e inferiores a los $1000^{\circ} \mathrm{C}$. Los tipos I y III (originales) reflejan temperaturas de cocción bajas, que oscilan entre los 700 y $900^{\circ} \mathrm{C}$. El tipo II (original) muestra una temperatura de cocción alta que ronda los $900-1000^{\circ} \mathrm{C}$. Los tipos IV y V, reflejan temperaturas de cocción medias de entre 800 y $900^{\circ} \mathrm{C}$.

- La similitud entre los ladrillos originales y los de sustitución se ve afianzada por los valores cromáticos. Esta similitud en el color indica una cuidada selección de las materias primas a la hora de fabricar los ladrillos de sustitución.

- La baja velocidad de propagación de los ultrasonidos es similar para los cinco tipos, pero junto con los índices de anisotropía, se detectan diferencias entre los tipos I y V, lo que indica que son ladrillos fácilmente deteriorables. Los altos índices de anisotropía, que presentan los cinco tipos de ladrillos, reflejan la presencia de importantes heterogeneidades, debidas posiblemente a los procesos de fabricación y que favorecen los procesos de deterioro; aunque para algunos de estos ladrillos (tipo V) haya pasado un corto periodo de tiempo desde su puesta en obra.

- En general, la porosidad de todos los ladrillos es alta $(>30 \%)$, destacando la porosidad de los tipos más modernos (IV y V), y seguidos de cerca por los más antiguos (tipo I).

- Los ladrillos de tipo I y los de tipo IV son muy similares desde el punto de vista porosimétrico y se caracterizan por tener una microporosidad dominante, una distribución porosimétrica unimodal $(1,5-2 \mu \mathrm{m})$, un tamaño de poro muy reducido y una superficie específica muy elevada.

\section{AGRADECIMIENTOS}

La investigación llevada a cabo en el Patrimonio de Talamanca de Jarama se ha realizado gracias a un convenio con la Dirección General de Patrimonio Histórico de la Comunidad de Madrid. Del mismo, agradecemos al Ayuntamiento de Talamanca de Jarama las facilidades aportadas para la realización de este trabajo. También agradecemos el apoyo recibido por la Comunidad de Madrid a través del proyecto MATERNAS_CM,0505/MAT/0094.

\section{BIBLIOGRAFÍA}

1. M.J. Rubio, P. López del Álamo, Talamanca de Jarama: fortificación y defensa, Editorial Valdés . Madrid (1990).

2. L. Torres Balbás, Talamanca y la ruta olvidada del Jarama, Boletín "La tierra, un material de construcción". Instituto Eduardo Torroja. Madrid. 77-82 (1960).

3. M.C. Hermida, L. Gómez y A.I. Arévalo, Talamanca del Jarama en la Edad Media, Consejo Superior de Investigaciones Científicas, Madrid. (1991).

4. M.J. Landete, Los restos arqueológicos de Salamanca del Jarama, Revista de Arqueología, 18, 6-9 (1982).

5. F. Saez-Lara, Catálogo de los Castillos, fortificaciones y recintos amurallados medievales de la Comunidad de Madrid, 275 (1993).

6. J. Warren, Conservation of Brick, Butterworths/Heinemann, Oxford (1999).

7. P. Cardiano, S. Ioppolo, C. Stefano, A. Pettignano, S. Sergi, P. Piraino, Study and chatacterization of the ancient bricks of monastery of "San Filippo di Fragalà" in Frazzanò (Sicily), Analytica Chimica Acta 519, 103-111 (2004).

8. J.Capel, F. Huertas, J. Linares. «High temperature reactions and use of Bronze Age pottery from La Mancha central Spain». Miner. Petrogr. Acta 29A, 563-575. (1985).9. M.P. Riccardi, B. Messiga, P. Duminuco, An approach to the dynamics of clay firing, Appl. Clay. Sci. 15, 393-409 (1999).

10. S. Shoval. «The firing temperature of a Persian-period pottery kiln at TelMichal, Israel, estimated from the composition if its pottery». J. Thermal Anal. 42, 1, 175-185 (1994).

11. R. Muñoz, J. Capel, E. Reyes, Hidrólisis de materiales cerámicos. Estudio Preliminar, Bol. Soc. Esp. Min. 13, 1, 43-44 (1990).

12. S. Caro Calatayud, S. Pavía Santamaría, F. Pérez Lorente, Influencia de la paragénesis mineral, porosidad y defectos estructurales en la alterabilidad de los ladrillos macizos antiguos, Geogaceta 30, 31-33 (2001).

13. R. Fort, M. Álvarez de Buergo, M.C. López de Azcona, F. Mingarro, MJ Varas, J. Soriano, Caracterización de la Fábrica de Ladrillo del Palacio del Infante Don Luis, Boadilla de Monte, Madrid, Bol. Soc. Esp. Ceram. V., 43 [2] 578-582 (2004).

14. R.W. Richards, Brick manufacturing from past to present, Ceram Bull, 69, 5, 807-813 (1990).

15. P. López Arce, J. García Guinea, M. Gracia, J. Obis, Bricks in historical buildings of Toledo City: characterization and restoration, Materials Characterization, 50, 59-68 (2003).

16. P. López Arce, J. García Guinea, Weathering traces in ancient bricks from historic buildings, Building and Environment, 40, 929-941 (2005).

17. Commission Internationale de $l^{\prime}$ Eclairage (CIE) «Colorimetry» $2^{\text {nd }}$ ed, Publication CIE 15.2, Bureau central de la CIE. Paris, 83 pp. (1986).

18. R.C. Mackenzie, Diferencial Thermal Analysis, Vol I. Academia Press. London and New York. (1970).

19. G. Cultrone, E. Sebastián, K. Elert, M.J. de la Torre, O. Cazalla, C. Rodróguez-Navarro., Influence of mineralogy and firing temperaturas in the porosity of bricks, J. Europ. Ceram. Soc. 24, 547-564 (2004).

20. S.R. Mallidi, Application of mercury intrusion porosimetry on clay bricks to asses freeze-thaw durability, A bibliography with abstracts. Constr. Build. Mater., 10, 6, 461-465 (1996).

Recibido: 22.12 .05

Aceptado: 23.05.06 